\title{
Measurement of Phase Space Density Evolution in MICE
}

\author{
Moses Chung* \\ Ulsan National Institute of Science and Technology \\ E-mail: mchung@unist.ac.kr
}

\section{François Drielsma ${ }^{\dagger}$}

University of Geneva

E-mail: francois.drielsma@unige.ch

\begin{abstract}
The Muon Ionization Cooling Experiment (MICE) collaboration has demonstrated the feasibility of ionization cooling, the technique by which it is proposed to cool the muon beam at a future neutrino factory or muon collider. The position and momentum reconstruction of individual muons in the MICE trackers allows for the development of alternative figures of merit in addition to beam emittance. Contraction of the phase space volume occupied by a fraction of the sample, or equivalently the increase in phase space density at its core, is an unequivocal cooling signature. Single-particle amplitude and nonparametric statistics provide reliable methods to estimate the phase space density function. These techniques are robust to transmission losses and non-linearities, making them optimally suited to perform a quantitative cooling measurement in MICE. Preliminary results are presented.
\end{abstract}

The 39th International Conference on High Energy Physics (ICHEP2018)

4-11 July, 2018

Seoul, Korea

${ }^{*}$ Speaker.
${ }^{\dagger}$ Corresponding author.

(C) Copyright owned by the author(s) under the terms of the Creative Commons 
Ionization cooling [1] is the only practical technique to cool muon beams at a future Neutrino Factory or Muon Collider [2]. MICE [3] has taken data in the Step IV configuration in order to make detailed measurements of the phase space evolution of muons through an absorber. Results from one transfer line configuration are reported [4]. The accumulated muon sample had a nominal emittance of $6 \mathrm{~mm}$ for a mean momentum of $140 \mathrm{MeV} / \mathrm{c}$ in the upstream tracker. The absorber was a single $65 \mathrm{~mm}$-thick lithium hydride disk placed at a $\beta_{\perp} \simeq 520 \mathrm{~mm}$ focus.

The 4D amplitude of a particle with phase space vector $\mathbf{v}=\left(x, p_{x}, y, p_{y}\right)$ is given by

$$
A_{\perp}=\varepsilon_{\perp}(\mathbf{v}-\boldsymbol{\mu})^{T} \Sigma^{-1}(\mathbf{v}-\boldsymbol{\mu}) .
$$

with $\varepsilon_{\perp}$ the transverse RMS emittance and $\boldsymbol{\mu}=\left(\langle x\rangle,\left\langle p_{x}\right\rangle,\langle y\rangle,\left\langle p_{y}\right\rangle\right)$, the beam centroid. The $\alpha$ subemittance, $e_{\alpha}$, is defined as the RMS emittance of the high-amplitude core fraction $\alpha$ of the parent beam. If the same amount of particles are selected upstream and downstream, the relative subemittance change is identical to that of the normalized RMS emittance [4]. The evolution of the $9 \%$-subemittance is represented in figure 1 and shows a clear cooling signal.

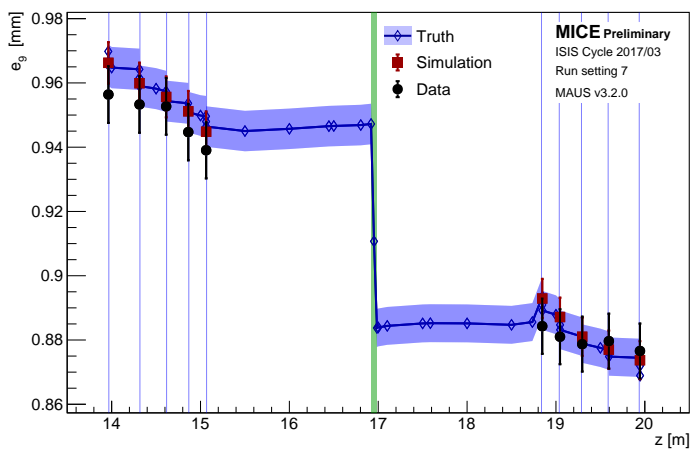

Figure 1: $9 \%$-subemittance evolution through the MICE cooling channel.



Figure 2: $9^{\text {th }}$ percentile $k \mathrm{NN}$ density level evolution through the MICE cooling channel.

Nonparametric density estimators were also investigated and systematic studies showed that the $k$-Nearest Neighbour $(k \mathrm{NN})$ algorithm is the most efficient and robust method in four dimensions [4]. For a given phase space vector $\mathbf{v}=\left(x, p_{x}, y, p_{y}\right)$, find the $k$ nearest points in the $n$ points cloud, calculate the distance to the $k^{\text {th }}$ nearest neighbor, $R_{k}$, and evaluate the density as

$$
\rho(\mathbf{v})=k n^{-1} V_{k}^{-1}=2 k n^{-1} \pi^{-2} R_{k}^{-4},
$$

with $V_{k}$ the volume of the 4-ball of radius $R_{k}$. The evolution of the density function's $9^{\text {th }}$ percentile is represented in figure 2 . This quantity experiences a fractional increase consistent with the squared inverse relative change in emittance, as expected [4].

\section{References}

[1] D. Neuffer, Part. Accel. 14 (1983) 75-90

[2] M. Alsaharo'a et al., Phys. Rev. ST Accel. Beams 6 (2003) 081001

[3] http://mice.iit.edu/

[4] F. Drielsma, PhD thesis (2018) University of Geneva, 